Check for updates

Cite this: Phys. Chem. Chem. Phys., 2020, 22, 9308

Received 20th January 2020 Accepted 7th April 2020

DOI: $10.1039 / d 0 c p 00335 b$

rsc.li/pccp

\title{
Phase separation in pore-spanning membranes induced by differences in surface adhesion $\dagger$
}

\author{
Jeremias Sibold, ${ }^{a}$ Vera E. Tewaag, ${ }^{a}$ Thomas Vagedes, ${ }^{a}$ Ingo Mey ${ }^{a}$ and \\ Claudia Steinem (D) *ab
}

\begin{abstract}
Lipid domains in plasma membranes act as molecular sorting platforms for e.g., signalling processes. In model membranes, such as freestanding or supported bilayers, some lipid domains with defined chemical composition, lipid packing and physical behaviour can be reproduced. However, in vivo, the plasma membrane experiences a proteinaceous scaffold underneath, which can sort, compartmentalize and recruit components within the membrane. The influence of such scaffolds on the phase behaviour of lipid membranes has been barely studied. Here, we investigated the partial attachment of a membrane to a support and its influence on the phase behaviour using pore-spanning membranes (PSMs). PSMs were prepared on $\mathrm{SiO}_{x=1-2}$ functionalized silicon substrates with $1.2 \mu \mathrm{m}$-sized pores by spreading giant unilamellar vesicles (GUVs) composed of DOPC/sphingomyelin (1:1) with different cholesterol concentrations. Using two different fluorophores, PSMs were visualized by fluorescence microscopy allowing us to distinguish between different membrane phases, a gel $\left(l_{\beta}\right)$, a liquid ordered $\left(l_{0}\right)$, and a liquid disordered $\left(l_{\mathrm{d}}\right)$ phase. At low cholesterol concentrations, coexistence of $l_{\beta}$ and $l_{\mathrm{d}}$ was found, while at higher cholesterol concentrations, coexistence of $l_{\circ}$ and $l_{d}$ was predominant. Below the mixing temperature, determined by temperature scans, the more ordered phase was always found in the freestanding PSMs, whereas the $l_{\mathrm{d}}$-phase was present in the supported PSMs. We attribute this lipid sorting to a stronger adhesion of the $l_{d}$-phase lipids to the underlying scaffold. The difference in adhesion alters the phase behaviour from a nominal DOPC/sphingomyelin (1:1) mixture to a DOPC/ sphingomyelin (1:2-1:4) mixture compared to phase diagrams obtained from GUVs highlighting the importance of differential adhesive surfaces on lipid domain formation.
\end{abstract}

\section{Introduction}

The organisation of the plasma membrane of eukaryotic living cells crosses length scales of a few nanometres to several micrometres. The function of the plasma membrane i.e., the formation of a dielectric barrier to the outside and the exchange of information with the environment heavily relies on the participating lipids and their spatiotemporal organisation. Since the seminal paper of Simons and Ikonen, ${ }^{1}$ particular lipid domains within the plasma membrane, so-called 'lipid rafts' have gained superior attention. ${ }^{2}$ A plethora of studies has been published elucidating the dynamic heterogeneity of raft domains in vitro and in vivo. ${ }^{3}$ In vivo, raft domains are described as highly dynamic structures with diameters of 10-200 nm

\footnotetext{
${ }^{a}$ Institute of Organic and Biomolecular Chemistry, University of Göttingen, Tammannstr. 2, 37077 Göttingen, Germany. E-mail: csteine@gwdg.de

${ }^{b}$ Max Planck Institute for Dynamics and Self-Organization, Am Fassberg 17, 37077 Göttingen, Germany

$\dagger$ Electronic supplementary information (ESI) available. See DOI: 10.1039/ d0cp00335b
}

enriched in sphingolipids and cholesterol. ${ }^{4}$ They are discussed to be involved in cellular processes like signalling, apoptosis, protein organisation, endocytosis and adhesion. ${ }^{1,5,6}$ Owing to their small size and their dynamics, they are however difficult to visualize in vivo. ${ }^{7}$

Hence, the majority of studies was performed using artificial membranes such as giant unilamellar vesicles (GUVs), multilamellar vesicles or supported lipid bilayers (SLBs), with lipid compositions resembling the outer leaflet of the plasma membrane. These membranes are composed of ternary mixtures of a high melting lipid such as sphingomyelin (SM) or 1,2-dipalmitoyl-sn-glycero-3-phosphocholine (DPPC) and cholesterol (Chol) and a low melting lipid such as 1,2-dioleoylsn-glycero-3-phosphocholine (DOPC) or 1-palmitoyl-2-oleoyl-snglycero-3-phosphocholine (POPC). ${ }^{4,8-13}$ Phase diagrams of such ternary lipid mixtures have been obtained for GUVs by means of fluorescence microscopy, ${ }^{14,15}$ for multilamellar vesicles using NMR spectroscopy, ${ }^{16}$ while SLBs were investigated by atomic force microscopy. ${ }^{8}$ The obtained ternary phase diagrams show a coexistent region of two liquid phases, a liquid-ordered $\left(l_{\mathrm{o}}\right)$ - and a liquid-disordered $\left(l_{\mathrm{d}}\right)$-phase. The $l_{\mathrm{o}}$-phase is enriched in 
Chol and the high melting temperature lipid, while the $l_{\mathrm{d}}$-phase is enriched in the low melting temperature lipid. As an example, the composition of the $l_{\mathrm{o}}$ - and $l_{\mathrm{d}}$-phase for the typical phase-separated DOPC/SM/Chol (2:2:1) mixture with $\mathrm{SM}_{\text {egg }}$ has been calculated using the ternary phase diagram obtained from GUVs with tie lines ${ }^{17}$ to be $l_{\mathrm{o}}\left(\mathrm{DOPC} / \mathrm{SM}_{\text {egg }} / \mathrm{Chol}\right)=$ 9.5:55:35.5 $(n / n)$ and $l_{\mathrm{d}}\left(\mathrm{DOPC} / \mathrm{SM}_{\mathrm{egg}} / \mathrm{Chol}\right)=70.5: 25: 4.5$ $(n / n)$. The $l_{\mathrm{d}}$-phase has loose lateral lipid packing, acyl chains with gauche kinks, and fast lateral diffusion. In contrast, the $l_{\mathrm{o}}$-phase is characterised by tight lipid packing and a high degree of order, but still significant lateral diffusion. ${ }^{18}$ Even though $l_{\mathrm{o}}$-domains have similarities to raft domains, their size and physical properties are different from the domains found in the plasma membrane. ${ }^{7,10}$

One significant difference between the in vivo situation and artificial membranes is the fact that in model membranes, such as GUVs or SLBs, the bilayer is either completely freestanding (GUVs) or entirely attached to a solid substrate (SLBs). However, in vivo the plasma membrane is only partly freestanding. A large part of the membrane is linked to other cell structures such as cell-cell junctions, focal adhesions, and the cytoskeleton. ${ }^{19}$ These linkages greatly influence the mobility and organisation of the membrane components as shown by Schneider et al. ${ }^{20}$ They found that the diffusion of phospholipids and sphingolipids is considerably hindered in the plasma membranes of cells, while in giant plasma membrane vesicles (GPMVs) derived from these cells, the diffusion was unhindered due to the missing actin cytoskeleton.

To investigate the impact of the cytoskeleton on the phase behaviour of GUVs, Liu et al. ${ }^{21}$ polymerized an F-actin network on the GUV membranes inducing phase separation of initially homogenous vesicles. Honigmann et $a{ }^{22}{ }^{22}$ pinned an F-actin network to $l_{\mathrm{o}} / l_{\mathrm{d}}$-phase-separated SLBs via streptavidin-biotin linkages, which significantly altered the lipid domain structure. Along these lines, several groups investigated the influence of an underlying pre-patterned substrate on the phase behaviour of phase-separated SLBs. ${ }^{23}$ Patterning was either realized physically using different surface topographies ${ }^{24-26}$ or chemically with different polymer coatings. By using a patterning of polymers with either a saturated or an unsaturated lipid, the binding of particular lipids to the surface was altered leading to the recruitment of certain membrane components. ${ }^{27,28}$ Alternatively, Morigaki and coworkers ${ }^{29-31}$ used polymers that were partially or fully removed in confined areas to control membrane adhesion to the surface. They found that the $l_{\mathrm{o}}$-phase is localized in areas without polymer coating and the $l_{\mathrm{d}}$-phase in areas with partial polymer coating. The results demonstrate that the differential adhesion of the membrane to a support alters its phase behaviour. These findings imply that not only the membrane but its local adhesion to the cytoskeleton considerably impacts the lateral organisation of the plasma membrane in vivo. However, compared to SLBs, the plasma membrane is composed of freestanding bilayer areas with an only partial attachment to the underlying scaffold.

To mimic such situation, a membrane model, where a bilayer only partially experiences a support is highly desirable and can be generated by using pore-spanning membranes (PSMs). ${ }^{32}$ In this system, a membrane with an underlying nano- to micrometre-sized scaffold providing freestanding (f-PSMs) as well as supported (s-PSMs) bilayer areas is generated in a planar geometry readily accessible by fluorescence microscopy. ${ }^{19,33-42}$ As yet, PSMs composed of PC/SM/Chol have been formed on gold functionalized pore rims, and hence, owing to the quenching of the fluorescence by the gold layer, s-PSMs could not be fluorescently observed lacking the information about $l_{\mathrm{o}} / l_{\mathrm{d}}$-phase separation of the entire PSM. ${ }^{43,44}$ To overcome this drawback, we here make use of $\mathrm{SiO}_{x=1-2}$ functionalized porous silicon substrates ${ }^{33}$ in combination with two fluorescent $\left(l_{\mathrm{d}^{-}}\right.$and $\left.l_{\mathrm{o}}-\right)$ markers allowing the visualization of f-PSMs and s-PSMs. Our results on DOPC/ SM/Chol PSMs with different Chol contents highlight the importance of the differences in adhesion of particular membrane lipids considerably altering the phase behaviour.

\section{Materials and methods}

\section{Materials}

1,2-Dioleoyl-sn-glycero-3-phosphocholine (DOPC), sphingomyelin from porcine brain $\left(\mathrm{SM}_{\text {porc }}\right.$, the fatty acid distribution can be found in the ESI $\dagger$ ), and TopFluor cholesterol (BODIPY-Chol) were purchased from Avanti Polar Lipids (Alabaster, AL, USA). Cholesterol (Chol) and sulforhodamine-1,2-dihexanoyl-snglycero-3-phosphoethanolamine (TexRed-DHPE) were obtained from Sigma-Aldrich (Taufkirchen, Germany). Silicon monoxide granules (4-8 mm) were purchased from Merck (Darmstadt, Germany). Porous silicon nitride substrates were received from Aquamarijn (Zutphen, The Netherlands).

\section{Preparation of giant unilamellar vesicles (GUVs)}

Phase-separated liquid ordered $\left(l_{\mathrm{o}}\right)$ /liquid disordered $\left(l_{\mathrm{d}}\right)$ GUVs were prepared by the electroformation method. Lipids dissolved in chloroform $(0.1 \mathrm{mg})$ were deposited on preheated indium tin oxide coverslips and the organic solvent was removed under reduced pressure at $55{ }^{\circ} \mathrm{C}$. The molar ratio of the lipids was DOPC/SM $\mathrm{Sorc}_{\text {chol }} / \mathrm{Ch}$ different Chol molar concentrations ranging from $n_{\mathrm{Chol}}=0-0.5\left(\mathrm{DOPC} / \mathrm{SM}_{\mathrm{porc}} / \mathrm{Chol}, 0.5-\left[n_{\mathrm{Chol}} / 2\right]\right.$ : $\left.0.5-\left[n_{\mathrm{Chol}} / 2\right]: n_{\mathrm{Chol}}\right)$. To label the $l_{\mathrm{d}}$-phase, $0.5 \%$ of DOPC was replaced by TexRed-DHPE and to label the $l_{\mathrm{o}}$-phase, $0.5 \%$ of Chol was replaced by BODIPY-Chol. Electroformation was performed in sucrose solution ( $2 \mathrm{~mL}, 300 \mathrm{mM})$, applying a sinusoidal voltage of $1.6 \mathrm{~V}$ and $12 \mathrm{~Hz}$ at $55{ }^{\circ} \mathrm{C}$ for 2.5 hours. GUVs were stored at room temperature for up to 3 days.

\section{Functionalization of porous substrates}

Porous silicon nitride substrates with a pore radius of $0.6 \mu \mathrm{m}$ were functionalized with a $30 \mathrm{~nm} \mathrm{SiO}_{x=1-2}$-layer. $\mathrm{SiO}$ was evaporated at a deposition rate of $0.3 \mathrm{~nm} \mathrm{~s}^{-1}$ on top of the substrates (Bal-Tec Med020; Oerlikon Balzers, Liechtenstein). Before use, the substrates were stored in a $55{ }^{\circ} \mathrm{C}$ heated water bath for $1 \mathrm{~h}$ resulting in a mixture of $\mathrm{SiO}$ and $\mathrm{SiO}_{2}{ }^{33}$

\section{Preparation of pore-spanning membranes (PSMs)}

The functionalized substrates were rinsed with ethanol, ultra-

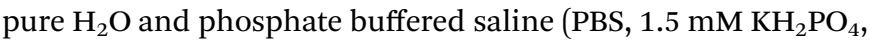


$8.1 \mathrm{mM} \mathrm{Na} \mathrm{HPO}_{4}, 2.7 \mathrm{mM} \mathrm{KCl}, 136 \mathrm{mM} \mathrm{NaCl}, \mathrm{pH}$ 7.4). After rinsing, they were placed into a PBS-filled $(1 \mathrm{~mL})$ temperaturecontrolled chamber ${ }^{45}$ and heated up to $55^{\circ} \mathrm{C}$. $75 \mu \mathrm{L}$ of the GUV solution were added through a $5 \mathrm{~mL}$ pipette tip filled with PBS at $55{ }^{\circ} \mathrm{C}$. This procedure partially separates the GUVs filled with sucrose from other lipid material. After $10 \mathrm{~min}$ of incubation, PSMs have been formed.

\section{Fluorescence microscopy of PSMs}

PSMs were analysed with a confocal laser scanning microscope (FV 1200 Olympus, Hamburg, Germany) equipped with a cooled GaAsP-PMT detector and a water immersion objective (LPUMPlan N $60 \times / 1.0$ NA Olympus, Hamburg, Germany). BODIPY-Chol was excited at $\lambda_{\text {ex }}=488 \mathrm{~nm}$ and emission was collected with a band-pass filter from $\lambda_{\mathrm{em}}=505-540 \mathrm{~nm}$. TexRed-DHPE was excited at $\lambda_{\mathrm{ex}}=561 \mathrm{~nm}$ and the emission was collected with a band-pass filter from $\lambda_{\mathrm{em}}=575-675 \mathrm{~nm}$. Each image was taken within $4.36 \mathrm{~s}$ (filter mode: Kalman $2 \times$ line). The presented fluorescence images were processed with a mean $3 \times 3$ filter. For the temperature-dependent measurements, $T$ was adjusted between $25-55{ }^{\circ} \mathrm{C}$ with a heating or cooling velocity of $0.2 \mathrm{~K} \mathrm{~s}^{-1}$. Once the temperature was constant, the membrane patch was equilibrated for $1 \mathrm{~min}$ prior to image acquisition. Heating and cooling cycles were performed.

From the fluorescence micrographs, temperature dependent curves were obtained, from which the mixing temperature $T_{\mathrm{M}}$ was extracted. A detailed description of the analysis of the fluorescence micrographs of PSMs as a function of temperature is given in the ESI $\dagger$ (Fig. S1 and S2).

\section{Results and discussion}

To investigate the phase behaviour of liquid ordered $\left(l_{\mathrm{o}}\right) /$ liquid disordered $\left(l_{\mathrm{d}}\right)$ coexisting phases in membranes on porous substrates dependent on the Chol content and temperature, PSMs were generated on $\mathrm{SiO}_{x=1-2}$ functionalized substrates by GUV spreading. GUVs composed of a $1: 1$ mixture of DOPC and $\mathrm{SM}_{\text {porc }}$ with various amounts of Chol (0-50 mol\%) were spread on porous silicon substrates with $0.6 \mu \mathrm{m}$ pore radius and a surface porosity of $39-44 \%$ (Fig. S3, ESI $\dagger$ ) at $55{ }^{\circ} \mathrm{C}$. Afterwards, the PSMs were cooled down slowly to room temperature to reach equilibrium conditions. This procedure is different from a study, where we used phase-separated PSMs on $\mathrm{SiO}_{x=1-2}$ functionalized porous surfaces obtained by rapid cooling resulting in kinetically trapped domains. ${ }^{33} \mathrm{~A}$ different procedure was also used by Sumitomo et al., ${ }^{38}$ who spread DOPC/ DPPC/Chol $(30: 40: 30)$ bilayers on porous $\mathrm{SiO}_{2}$ substrates with the help of $\mathrm{CaCl}_{2}$. They observed lipid domains in the $10 \mu \mathrm{m}$ regime with a partial change in fluorescence over time also suggesting a non-equilibrium state. We observed the obtained PSMs using two different fluorescent lipids. The $l_{\mathrm{d}}$-phase was visualized with sulforhodamine-1,2-dihexanoyl-sn-glycero3-phosphoethanolamine (TexRed-DHPE, $l_{\mathrm{d}}$-phase marker) ${ }^{46,47}$ and the $l_{\mathrm{o}}$-phase with TopFluor cholesterol (BODIPY-Chol, $l_{\mathrm{o}}$-phase marker). ${ }^{47,48}$ The phase behaviour of the PSMs was
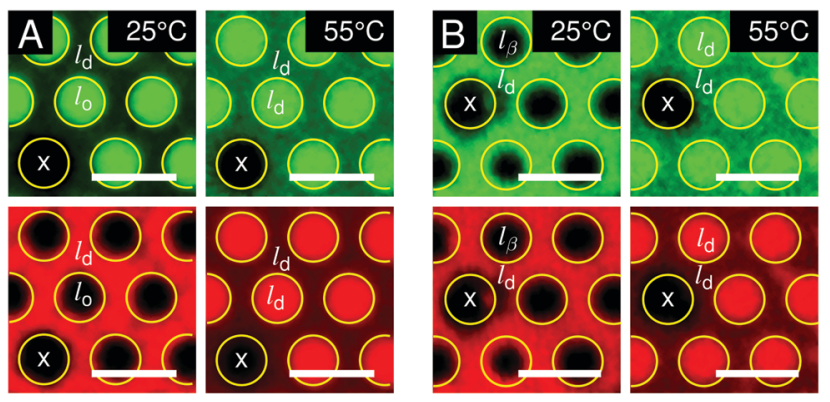

Fig. 1 Fluorescence micrographs of PSMs at $25{ }^{\circ} \mathrm{C}$ and $55^{\circ} \mathrm{C}$. Images in the upper row show the BODIPY-Chol fluorescence, while images in the lower row show the TexRed-DHPE fluorescence. (A) PSMs composed of DOPC/SM pord $/ C h o l(41.5: 41.5: 17)$. (B) PSMs composed of DOPC/SM pord Chol $(50: 50: 0)$. The gel phase $l_{\beta}$, the liquid ordered phase $l_{\circ}$ and the liquid disordered phase $l_{d}$ are assigned to the f-PSM and s-PSM according to the partition of the two fluorophores TexRed-DHPE and BODIPY-Chol. The pore structure of the underlying substrate is given by yellow circles. An uncovered pore is marked by an x. Scale bars: $2 \mu \mathrm{m}$.

observed by fluorescence microscopy at different temperatures covering the range $\left(T=25-55{ }^{\circ} \mathrm{C}\right)$ in which mixing/demixing of the membranes is expected.

Fig. 1 shows typical fluorescence micrographs of PSMs with BODIPY-Chol (green) and TexRed-DHPE (red) at $T=25{ }^{\circ} \mathrm{C}$ and $T=55{ }^{\circ} \mathrm{C}$. In all images the regions of the freestanding PSMs (f-PSMs) can be distinguished from the supported PSMs (s-PSMs). The background intensity $I_{\text {Background }}$ (ESI $\dagger$ ) was determined from an uncovered pore, which is indicated with " $x$ " in the images. In Fig. 1A, PSMs with a lipid composition of DOPC/SM $/ \mathrm{Sorc}_{\text {phol }} / \mathrm{Ch}$ $(41.5: 41.5: 17)$ are depicted. At $25{ }^{\circ} \mathrm{C}$, the PSM is phaseseparated into an $l_{\mathrm{o}}$-phase indicated by the green BODIPY-Chol fluorescence, while the $l_{\mathrm{d}}$-phase is visualized by the red TexRedDHPE dye. From the fluorescence images at $25{ }^{\circ} \mathrm{C}$ it can be concluded that the $l_{\mathrm{o}}$-phase is only found in the f-PSMs, while the s-PSMs are only composed of $l_{\mathrm{d}}$-phase. At $55{ }^{\circ} \mathrm{C}$, both fluorophores are co-localized and distributed equally in both parts of the membranes, the s-PSMs and f-PSMs, respectively. Overall, the fluorescence intensity of the s-PSMs is diminished compared to that of the f-PSMs owing to partial fluorescence quenching on the $\mathrm{SiO}_{x=1-2}$ surface, which can be readily seen if homogeneous pure DOPC membranes are formed on the porous substrates (Fig. S4, ESI $\dagger$ ) and which has been also shown previously for other non-phase separated lipid compositions. ${ }^{33}$

For the lipid composition DOPC/SM $\mathrm{Sorc}_{\text {chol }}$ (50:50:0), a different scenario is observed (Fig. 1B). While the membrane is in a completely mixed state at $55{ }^{\circ} \mathrm{C}$, two phases can be distinguished at $25{ }^{\circ} \mathrm{C}$. Again, the s-PSMs are only composed of $l_{\mathrm{d}}$-phase indicated by the red TexRed-DHPE fluorescence. However, the f-PSMs contain an area, in which both fluorophores are excluded, which can be readily visualized by an overlay of both fluorescence images (Fig. S5, ESI $\dagger$ ). Even though these areas appear black, the fluorescence images at $55{ }^{\circ} \mathrm{C}$ unambiguously show that f-PSMs are present (Fig. 1B, right). We assign these membrane areas lacking both fluorophores to a gel phase $\left(l_{\beta}\right) \cdot{ }^{47,49}$ From ternary phase diagrams of DOPC/SM/Chol, it is known that a gel phase occurs at low Chol and high SM concentrations..$^{10,47,50-54}$ 
As the three detectable phases were clearly assigned by the two different fluorophores, we next asked the question how the Chol content influences the phase behaviour of the PSMs. We systematically varied the Chol content in the membranes, while keeping the DOPC/SM ratio constant to $1: 1$. We determined the transition temperature $\left(T_{\mathrm{M}}\right)$ of mixing/demixing by monitoring fluorescence images as a function of temperature. The TexRed-DHPE fluorescence intensity was used to extract quantitative information from the images, as TexRed-DHPE is known to partition to almost $100 \%$ in the $l_{\mathrm{d}}$-phase. ${ }^{46,47}$ In a previous study, we moreover analysed whether the chosen $l_{\mathrm{d}}$-phase marker alters the phase behaviour of ternary lipid mixtures, which turned out to be not the case. ${ }^{55}$ In contrast, labelling the $l_{\mathrm{o}}$-phase of model membranes is much more limited. We have chosen the well-studied BODIPY-Chol, which is known to partition into the $l_{\mathrm{o}}$-phase in model membrane systems with about $50-80 \% .^{3,48}$ Other fluorophores, which have been shown to partition in ordered phases of giant plasma membrane vesicles and plasma membranes partition, however in the $l_{\mathrm{d}}$-phase in model membrane systems. ${ }^{56-58}$ BODIPY-Chol was used to unambiguously distinguish between the $l_{\beta}$ - and $l_{\mathrm{o}}$-phase in the demixed state. However, owing to its only moderate partition in the $l_{\mathrm{o}}$-phase, it was not suited to determine the mixing temperature $T_{\mathrm{M}}$. To determine $T_{\mathrm{M}}$, the ratio of the TexRed-DHPE fluorescence intensities of the f-PSM ( $\left.I_{\mathrm{f}-\mathrm{PSM}}\right)$ and S-PSM $\left(I_{\text {S-PSM }}\right)$ were monitored. The analysis is described in detail in the ESI $\dagger$ (Fig. S1 and S2). The intensity ratios were plotted $v s$. temperature. To ensure that the system is in thermodynamic equilibrium, we performed heating-cooling cycles indicating only a small hysteresis (Fig. S6, ESI $\dagger$ ).

An example of a phase transition curve is shown in Fig. 2. A PSM composed of DOPC/SM porc $/ \mathrm{Chol}(46: 46: 8)$ is heated up from $25{ }^{\circ} \mathrm{C}$ to $55{ }^{\circ} \mathrm{C}$. The fluorescence micrographs (Fig. 2A and B) at $T=25{ }^{\circ} \mathrm{C}$ clearly indicate that both fluorophores are excluded from the f-PSMs meaning that the f-PSMs are in the $l_{\beta}$-phase (Fig. S7, ESI $\dagger$ ). The TexRed-DHPE fluorescence was observed in the s-PSMs representing the $l_{\mathrm{d}}$-phase. At $55{ }^{\circ} \mathrm{C}$ (Fig. 2A), a homogeneous distribution of the TexRed-DHPE fluorophores in the f-PSM and in the s-PSM was observed. Within a Chol concentration range of about $0-10 \mathrm{~mol} \%$, an $l_{\beta}$-phase was identified in the demixed membrane state. All corresponding $I_{\mathrm{f}-\mathrm{PSM}} / I_{\mathrm{S}-\mathrm{PSM}}(T)$ curves show a sigmoidal behaviour. A sigmoidal Boltzmann function (eqn (1)): ${ }^{59}$

$$
\frac{I_{\mathrm{f}-\mathrm{PSM}}}{I_{\mathrm{S}-\mathrm{PSM}}}(T)=\frac{I_{\mathrm{rel}, T=25^{\circ} \mathrm{C}}-I_{\mathrm{rel}, T=55^{\circ} \mathrm{C}}}{1+\exp \left(\frac{T-T_{\mathrm{M}}}{\mathrm{d} T}\right)}+I_{\mathrm{rel}, T=55^{\circ} \mathrm{C}}
$$

was fit to the data. $I_{\mathrm{rel}, T=25^{\circ} \mathrm{C}}$ and $I_{\mathrm{rel}, T=55^{\circ} \mathrm{C}}$ are the relative fluorescence intensities at 25 and $55{ }^{\circ} \mathrm{C}$, respectively; $\mathrm{d} T$ is the range in which the transition occurs and $T_{\mathrm{M}}$ the temperature at half-maximum of $I_{\mathrm{f} \text {-PSM }} / I_{\mathrm{S} \text {-PSM}}$. In case of the lipid composition shown in Fig. 2, a $T_{\mathrm{M}}=40.7{ }^{\circ} \mathrm{C}$ was determined (Fig. 2C). The phase separation near $T_{\mathrm{M}}$ is shown in an overlay of the two fluorophores (Fig. S7, ESI $\dagger$ ).

If the Chol concentration is increased above $10 \mathrm{~mol} \%$, the f-PSMs are composed of the $l_{\mathrm{o}}$-phase instead of the $l_{\beta}$-phase.

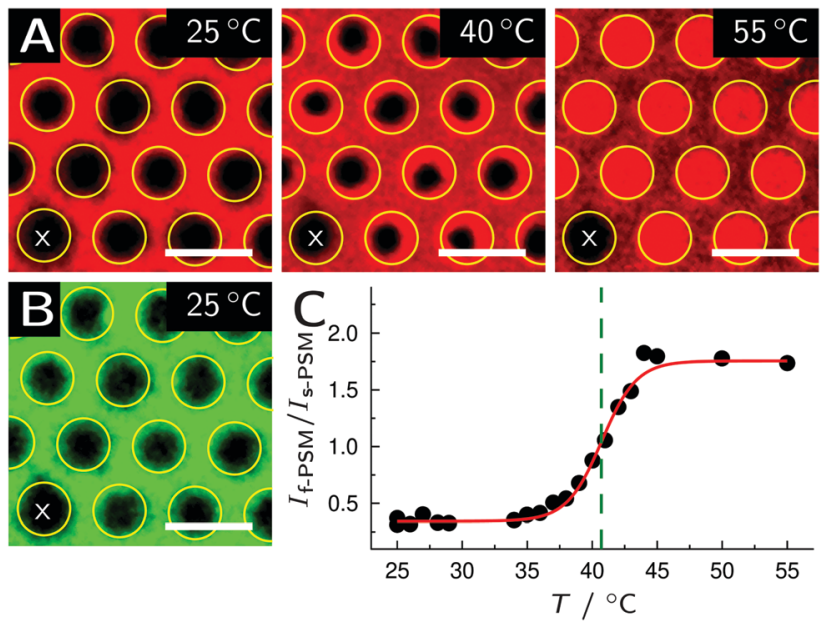

Fig. 2 Fluorescence micrographs of a PSM composed of DOPC/SM pord Chol $(46: 46: 8)$ obtained at different temperatures. The underlying porous array of the substrate is given by yellow circles and the non-membrane covered pore is marked with an $\mathrm{x}$. (A) The TexRed-DHPE fluorescence images at $T=25,40$ and $55{ }^{\circ} \mathrm{C}$ show the phase transition from an $l_{B} / l_{d}$-phaseseparated membrane to a homogeneous $l_{d}$-phase. (B) The BODIPY-Chol fluorescence image at $T=25^{\circ} \mathrm{C}$ indicates that the PSM phase-separates into an $l_{\beta}-$ and $l_{d}$-phase. Scale bars: $2 \mu \mathrm{m}$. (C) Relative fluorescence intensity as a function of temperature (black circles). Eqn (1) was fit to the data (red solid line) resulting in $\mathrm{d} T=1.35^{\circ} \mathrm{C}$ and in $T_{M}=40.7^{\circ} \mathrm{C}$ shown as green dashed line.

A PSM composed of DOPC/SM porc $_{\text {Chol }}(42.5: 42.5: 15)$ is shown in Fig. 3. At $25{ }^{\circ} \mathrm{C}$, TexRed-DHPE is excluded from the $\mathrm{f}$-PSMs, while BODIPY-Chol is enriched proving the existence of the $l_{\mathrm{o}}$-phase in the f-PSMs (Fig. $3 \mathrm{~A}$ and $\mathrm{B}, 25^{\circ} \mathrm{C}$ ). TexRed-DHPE fluorescence is observed in the s-PSMs labelling the $l_{\mathrm{d}}$-phase. From the corresponding $I_{\mathrm{f}-\mathrm{PSM}} / I_{\mathrm{s}-\mathrm{PSM}}(T)$ curve (Fig. $\left.3 \mathrm{C}\right), T_{\mathrm{M}}=$ $39.1{ }^{\circ} \mathrm{C}$ was obtained. Within a Chol concentration range of about $10-50 \mathrm{~mol} \%$ we always observed an $l_{\mathrm{o}}$ - and $l_{\mathrm{d}}$-phase in the demixed state.

Independent of whether the transition from $l_{\mathrm{o}} / l_{\mathrm{d}}$ to $l_{\mathrm{d}}$ or from $l_{\beta} / l_{\mathrm{d}}$ to $l_{\mathrm{d}}$ was observed, both phases, $l_{\beta} / l_{\mathrm{d}}\left(\right.$ Fig. $2 \mathrm{~A}, 40{ }^{\circ} \mathrm{C}$ ) or $l_{\mathrm{o}} / l_{\mathrm{d}}$ (Fig. 3A, $40{ }^{\circ} \mathrm{C}$ ) coexist in the f-PSMs during the transition. At this stage, $l_{\beta^{-}}$or $l_{\mathrm{o}}$-domains become discernible, even though the time resolution was not sufficient to resolve the circular appearance of such domains in the f-PSMs that we have visualized previously. ${ }^{44}$ Circular domains are indicative of a significant line tension, which has been determined by Schwille and coworkers for a similar lipid composition (DOPC/SM C $\mathrm{C}_{18: 0} / \mathrm{Chol}$, $2: 2: 1$ ) to be $1.2 \mathrm{pN}^{60}$ Tsai and Feigenson ${ }^{61}$ later demonstrated that an increase in the Chol content slightly reduces the line tension in such membrane mixtures. We found the described behaviour of the membranes for Chol concentrations of 0-18 mol\%. However, at larger Chol concentrations, the transition regime became substantially broader.

An example is given in Fig. 4 for a Chol concentration of $25 \mathrm{~mol} \%$. While the fluorescence micrographs still unambiguously indicate the presence of $l_{\mathrm{o}}$-and $l_{\mathrm{d}}$-phase at $25{ }^{\circ} \mathrm{C}$, no $l_{\mathrm{o}} / l_{\mathrm{d}}$ coexisting phase is observed in the f-PSMs during the phase transition (Fig. $4 \mathrm{~A}, 45{ }^{\circ} \mathrm{C}$ ). From the $I_{\mathrm{f}-\mathrm{PSM}} / I_{\mathrm{s}-\mathrm{PSM}}(T)$ curve (Fig. 4C), a $T_{\mathrm{M}}=45.7{ }^{\circ} \mathrm{C}$ was determined, even though it cannot 

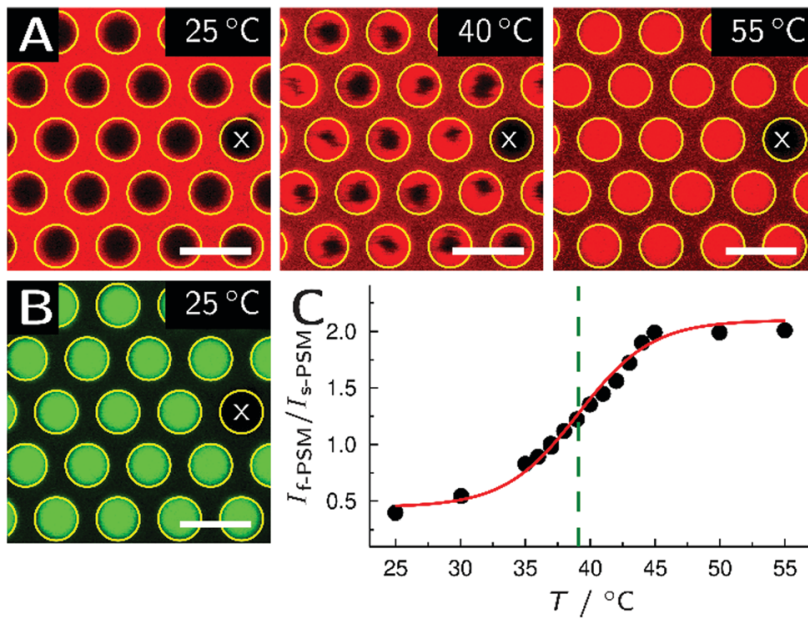

Fig. 3 Fluorescence micrographs of a PSM composed of DOPC/SM pord Chol $(42.5: 42.5: 15)$ obtained at different temperatures. The pores of the substrate are encircled in yellow and a non-membrane covered pore is marked with an $\mathrm{x}$. (A) The TexRed-DHPE fluorescence images at $T=25,40$ and $55{ }^{\circ} \mathrm{C}$ show the phase transition from the $l_{\mathrm{o}} / l_{\mathrm{d}}$-phase-separated membrane to a homogeneous $l_{d}$-phase. (B) The BODIPY-Chol fluorescence image at $T=25^{\circ} \mathrm{C}$ indicates that the PSM phase-separates into an $l_{\circ}$ and $l_{\mathrm{d}}$-phase. Scale bars: $2 \mu \mathrm{m}$. (C) Relative fluorescence intensity (black circles) as a function of temperature. Eqn (1) was fit to the data (red solid line) resulting in $\mathrm{d} T=2.85^{\circ} \mathrm{C}$ and in $T_{M}=39.1^{\circ} \mathrm{C}$, which is shown as green dashed line.

be ruled out that the transition is more complex and might contain a second non-resolved transition.

We analysed 22 different Chol concentrations. When the membrane is heated up or cooled down, it passes the transition temperature $T_{\mathrm{M}}$, which results in a coexistence of $l_{\mathrm{o}} / l_{\mathrm{d}^{-}}$or $l_{\beta} / l_{\mathrm{d}^{-}}$-phases within the PSMs below $T_{\mathrm{M}}$ independent of the lipid composition. The s-PSMs remain always in the $l_{\mathrm{d}}$-phase independent of temperature. The $T_{\mathrm{M}}$ values are plotted as a function of Chol concentration in a partial phase diagram (Fig. 5A). In a Chol range of 0-8 $\mathrm{mol} \%$, only a phase separation between $l_{\beta}$ - and $l_{\mathrm{d}}$-phase was found below $T_{\mathrm{M}}$. At a Chol concentration $\geq 11 \mathrm{~mol} \%$, only $l_{\mathrm{o}} / l_{\mathrm{d}}$-phase separation was detected. At Chol concentrations of 9-10 mol\%, both types of phase separation were observed. This uncertainty is probably a result of the compositional heterogeneity of the GUVs of up to $5 \%$ if obtained by electroformation. ${ }^{52,62-64}$ The largest Chol concentration under investigation was $50 \mathrm{~mol} \%$, which is still well below the maximum of soluble Chol that was determined to be $66 \mathrm{~mol} \%$ in DOPC membranes. ${ }^{65,66}$

Ternary phase diagrams composed of PC, SM and Chol have been determined for model membranes such as small vesicles and GUVs as well as for SLBs. ${ }^{67}$ In case of small vesicles and GUVs, the membranes do not experience any adhesion. However, if GUVs adhere with each other, and the system is driven towards the demixing transition, Gordon et al. ${ }^{68}$ found that phase separation invariably occurs first in the adhered region, which they discuss in terms of suppression of thermal shape fluctuations in these regions. In case of SLBs, the membranes experience a homogeneous adhesion, if the surface itself is not patterned, and membrane fluctuations are fully suppressed. In this study, membranes were prepared with two different
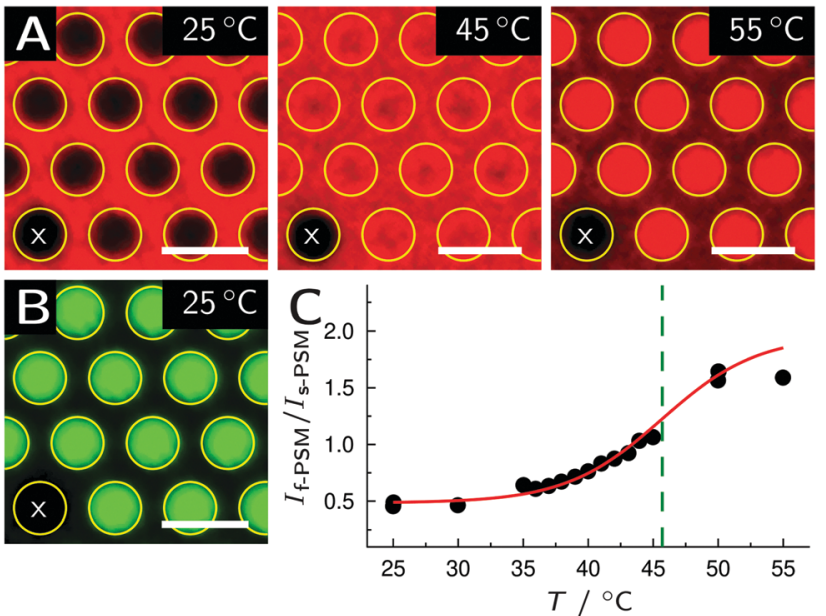

Fig. 4 Fluorescence micrographs of a PSM composed of DOPC/SM pord Chol $(37.5: 37.5: 25)$ obtained at different temperatures. The underlining porous array of the substrate is marked with yellow circles and a nonmembrane spanned pore is marked with an $\mathrm{x}$. (A) The TexRed-DHPE fluorescence images at $T=25,45$ and $55{ }^{\circ} \mathrm{C}$ demonstrate the phase transition from the $l_{0} / l_{d}$-phase-separated membrane to a homogeneous $l_{\mathrm{d}}$-phase, without a visualization of phase separation within the f-PSM. (B) The BODIPY-Chol fluorescence image at $T=25^{\circ} \mathrm{C}$ indicates that the PSM phase-separates into an $l_{\circ}$ and $l_{\mathrm{d}}$-phase. Scale bars: $2 \mu \mathrm{m}$. (C) Relative fluorescence intensity (black circles) as a function of temperature. Eqn (1) was fit to the data (red solid line) resulting in $\mathrm{d} T=3.70{ }^{\circ} \mathrm{C}$ and in $T_{\mathrm{M}}=$ $45.7^{\circ} \mathrm{C}$ shown as green dashed line.
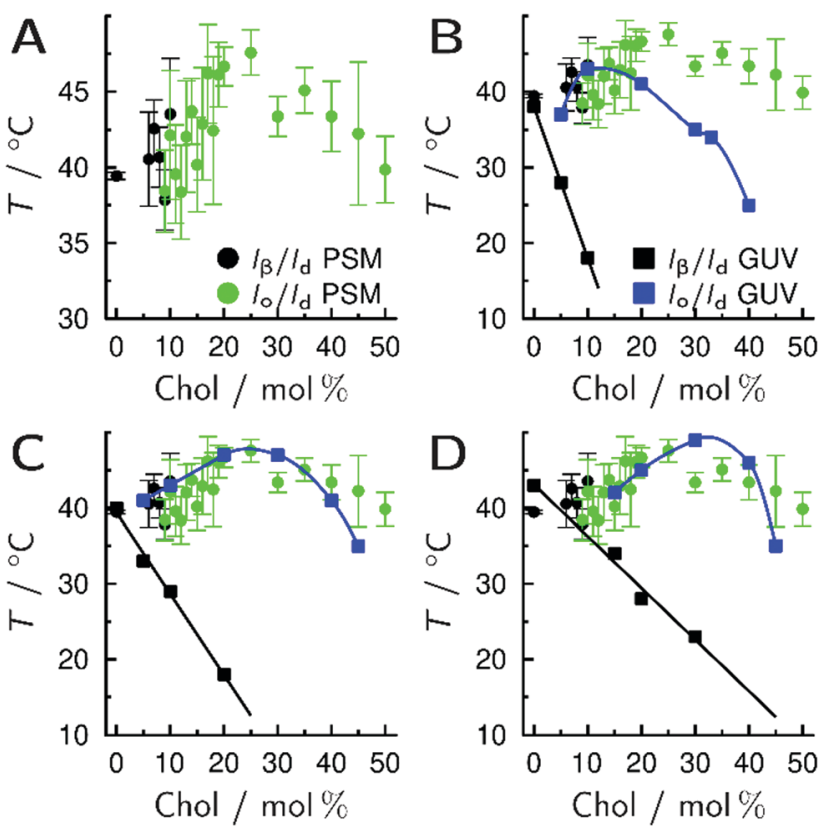

Fig. 5 (A) Partial phase diagram for PSMs composed of DOPC/SM porcl Chol with a constant ratio of DOPC and SMporc $(1: 1)$. Black symbols show the mean $T_{M}$ values of the $l_{\beta} / l_{d}$ to $l_{d}$ transitions and green symbols are the mean $T_{M}$ values for the $l_{0} / l_{d}$ to $l_{d}$ transitions. The error bars represent the standard deviation of the mean values. Comparison of the phase boundaries obtained for PSMs with those obtained for GUVs for a lipid mixture of (B) DOPC/SM C $16: 0$ (1:1), (C) DOPC/SM C $16: 0$ (1:2) and (D) DOPC/SM C $16: 0$ (1: 4). Data were taken from Veatch and Keller. ${ }^{50}$ 
micrometre-sized areas, a freestanding membrane part, where the lipids do not experience adhesion to a substrate and a supported part, where the lipids are in contact to the $\mathrm{SiO}_{x=1-2}$ support. These different adhesion areas influence the distribution of the lipids in the entire membrane, i.e., lipid sorting as a function of surface adhesion energy occurs. Membrane fluctuations in the f-PSMs can be neglected as the lateral membrane tension is with about $1 \mathrm{mN} \mathrm{m}{ }^{-1}$ or larger $^{32,69,70}$ at least one to two orders of magnitude larger than lateral membrane tensions found in GUVs. ${ }^{71}$ The influence of surface adhesion on the distribution of lipids is most obvious in the example presented in Fig. 1A. Below $T_{\mathrm{M}}$, only $l_{\mathrm{d}}$-phase is observed in the s-PSMs, while there is only $l_{\mathrm{o}}$-phase in the f-PSMs.

Such reorganisation and preferential localization of certain lipid phases as a function of a difference in surface adhesion has been described previously by Sarmento et al. ${ }^{72}$ using surface adhered GUVs. Lipowsky et al. ${ }^{73,74}$ treated this phenomenon theoretically relating the phase diagram of a binary DPPC/Chol mixture to the surface adhesion energies (affinity contrast). Our observation clearly demonstrates that the interaction of the $l_{\mathrm{d}}$-phase lipids with the support is stronger than that of the $l_{\mathrm{o}}$-phase lipids and leads to lipid sorting as a function of a partial interaction with the support. Two aspects need to be considered to explain this finding.

By AFM indentation experiments, we have shown previously that, owing to the fact that PSMs experience two different adhesive surfaces, the adhesion strength of the lipids to the solid substrate (s-PSMs) greatly influences the lateral pre-stress in the f-PSMs. ${ }^{69}$ To investigate how the adhesion of the two different lipid types, $l_{\mathrm{o}}$-phase lipids and $l_{\mathrm{d}}$-phase lipids, influences the lateral membrane tension, which is the surface free energy per unit area, in the f-PSMs, we performed AFM indentation experiments as described previously. ${ }^{75,76}$ We indented f-PSMs composed of only DOPC mimicking the $l_{\mathrm{d}}$-phase and f-PSMs composed of $\mathrm{SM}_{\text {pord }} /$ Chol $(60: 40)$ resembling the $l_{\mathrm{o}}$-phase. For DOPC, a mean membrane tension of $10.5 \pm 4.3 \mathrm{mN} \mathrm{m}^{-1}$ was determined, while on $\mathrm{SM}_{\text {porc }} /$ Chol membranes a slightly lower mean membrane tension of $6.7 \pm 4.0 \mathrm{mN} \mathrm{m}^{-1}$ was found (Fig. S8, ESI $\dagger$ ). This result implies that the $l_{\mathrm{d}}$-phase lipids adhere more strongly to the $\mathrm{SiO}_{x=1-2}$ surface than the $l_{\mathrm{o}}$-phase lipids consistent with our observation that the $l_{\mathrm{d}}$-phase lipids are preferentially localized in the s-PSMs and the $l_{\mathrm{o}}$-phase lipids in the f-PSMs.

A second aspect that needs to be considered is the surface roughness of the $\mathrm{SiO}_{x=1-2}$ substrate. We determined a mean surface roughness of $\mathrm{rms}=0.49 \pm 0.03 \mathrm{~nm}$ by means of atomic force micrographs. ${ }^{33}$ Even though the surface roughness is in the sub-nanometre regime, the lipid bilayer has to adopt to the underlying structure. This process is energetically less costly in case of a membrane composed of $l_{\mathrm{d}}$-phase lipids as these membranes have a lower bending stiffness than membranes composed of $l_{\mathrm{o}}$-phase lipids. ${ }^{77-79}$ On the molecular scale, the more ordered structure of the individual lipid tails in the $l_{\mathrm{o}}$-phase will favour a position of the $l_{\mathrm{o}}$-phase lipids on the smooth water surface (f-PSMs) compared to the rough $\mathrm{SiO}_{x=1-2}$ substrate (s-PSMs).

If we compare the $l_{\mathrm{o}}$ fraction in GUVs calculated according to the ternary phase diagram provided by Bezylepkina et al. ${ }^{17}$ with that found in our PSMs (Fig. S9, ESI $\dagger$ ), which is basically determined by the surface porosity (Fig. S3, ESI $\dagger$ ), we clearly see that the $l_{\mathrm{o}}$ fraction in GUVs increases with increasing Chol content, while it remains constant in PSMs. However, we cannot rule out that nanometresized $l_{\mathrm{o}}$ domains are present in the s-PSMs, which are not resolvable by optical microscopy. We analysed the maximum fluorescence change $\left(I_{\mathrm{rel}, T=55^{\circ} \mathrm{C}}-I_{\mathrm{rel}, T=25^{\circ} \mathrm{C}}\right)$ for $l_{\mathrm{o}} / l_{\mathrm{d}}$-coexisting phases in PSMs and found that it decreases in the range of a Chol content $=10-50 \%$ (Fig. S9, ESI $\dagger$ ), which might indicate that nanometre-sized $l_{\mathrm{o}}$-phase domains with excluded TexRedDHPE dye are located in the s-PSMs diminishing the relative fluorescence intensity at $T=25{ }^{\circ} \mathrm{C}$.

Furthermore, we compare the observed partial phase diagram (Fig. 5A) obtained for a nominal DOPC/SM $\mathrm{Sorc}_{\text {pre }}(1: 1)$ with those obtained by Veatch and Keller $^{50}$ (Fig. 5B-D) derived from GUVs, which are freestanding membranes. They used a mixture composed of DOPC/SM with a synthetic SM $\mathrm{C}_{16: 0}$, while we used $\mathrm{SM}_{\text {porc }}$, which is mainly (70\%) composed of saturated SM with SM $\mathrm{C}_{18: 0}$ as the major component (49\%). $20 \%$ of the SM is a monounsaturated $\mathrm{SM} \mathrm{C}_{24: 1}$ (see ESI $\dagger$ ), which is known to prevent domain segregation. ${ }^{80}$ Even though the SM composition is different from the one used by Veatch and Keller, we clearly see a trend in the phase boundaries as a function of Chol concentration. The phase boundaries for a DOPC/SM ratio of $1: 1$ derived from GUVs do not match our results (Fig. 5B). If $\mathrm{SM} \mathrm{C}_{24: 1}$ significantly contributed to the phase transition temperatures $T_{\mathrm{M}}$, we would expect to see decreased $T_{\mathrm{M}}$ values instead of increased ones. A better match is found for DOPC/SM (1:2) (Fig. 5C) and DOPC/SM (1:4) (Fig. 5D) ratios. This finding suggests that lipid sorting occurs, resulting in a change in the DOPC/SM ratio. It agrees with the observation that the s-PSMs are enriched in fluid phase DOPC lipids. As the overall lipid composition is constant, the ratio of DOPC/SM is locally shifted to larger SM concentrations in the f-PSMs. ${ }^{53,81}$

Another observation is that the lipid mixtures with a Chol content of $50 \mathrm{~mol} \%$ are still phase-separated, which is not observed for freestanding membranes in GUVs. For example, by means of fluorescence resonance energy transfer experiments, Petruzielo et al. ${ }^{82}$ found phase separation in $\mathrm{DOPC} / \mathrm{SM}_{\mathrm{brain}}$ multilamellar vesicles with up to $40 \mathrm{~mol} \%$ of Chol. Carravilla et $a .^{83}$ visualized phase separation by Laurdan in GUVs also only up to $40 \mathrm{~mol} \%$ of Chol. The same limit was observed for membranes attached to a solid support. Lipid bilayers composed of DOPC/SM $\mathrm{Sgg}_{\mathrm{eg}} / \mathrm{Chol}$ fully adhered to a support showed phase separation up to a Chol content of $40 \mathrm{~mol} \%{ }^{8}$

Our results show that ternary phase diagrams derived from simple lipid compositions only partially reproduce the nanoscale heterogeneity of eukaryotic plasma membranes. It is the cytoskeletal scaffold underneath the membrane providing different adhesive areas that also contributes significantly to the phase behaviour of a lipid bilayer.

\section{Conclusions}

While a number of studies have explored the coexistence of lipid phases in model membrane systems, less attention has 
been drawn to an underlying patterned structure influencing the phase behaviour of such membranes. By using porespanning membranes, a well-defined nano- to micrometresized scaffold can be attached to a freestanding membrane allowing one to systematically investigate its influence on the phase behaviour of lipid bilayers. Even though this model system is still simplistic, it provides valuable information about organisation principles that control sorting and compartmentalization of lipids in biomembranes. Differences in adhesion of particular lipids within the membrane alters the phase behaviour considerably giving rise to a rich phase diagram with a variety of domain sizes with different physical properties. This is prerequisite for the dynamic interplay of lipid domains as they occur during signalling processes.

\section{Conflicts of interest}

There are no conflicts to declare.

\section{Acknowledgements}

Financial support by the Deutsche Forschungsgemeinschaft (Collaborative Research Center 803, project A05) is gratefully acknowledged. We further thank the VW-foundation for financial support. Open Access funding provided by the Max Planck Society.

\section{Notes and references}

1 K. Simons and E. Ikonen, Nature, 1997, 387, 569-572.

2 K. Simons and D. Toomre, Nat. Rev. Mol. Cell Biol., 2000, 1, 31-39.

3 E. Sezgin, I. Levental, S. Mayor and C. Eggeling, Nat. Rev. Mol. Cell Biol., 2017, 18, 361-374.

4 S. L. Veatch, O. Soubias, S. L. Keller and K. Gawrisch, Proc. Natl. Acad. Sci. U. S. A., 2007, 104, 17650-17655.

5 K. Simons and M. J. Gerl, Nat. Rev. Mol. Cell Biol., 2010, 11, 688-699.

6 K. Gaus, E. Gratton, E. P. W. Kable, A. S. Jones, I. Gelissen, L. Kritharides and W. Jessup, Proc. Natl. Acad. Sci. U. S. A., 2003, 100, 15554-15559.

7 F. M. Goñi, Chem. Phys. Lipids, 2019, 218, 34-39.

8 A. Aufderhorst-Roberts, U. Chandra and S. D. Connell, Biophys. J., 2017, 112, 313-324.

9 G. W. Feigenson, Biochim. Biophys. Acta, 2009, 1788, 47-52.

10 E. London, Biochim. Biophys. Acta, 2005, 1746, 203-220.

11 L. A. Bagatolli and O. G. Mouritsen, Front. Plant Sci., 2013, 4, 457.

12 L. J. Pike, J. Lipid Res., 2006, 47, 1597-1598.

13 J. H. Davis, J. J. Clair and J. Juhasz, Biophys. J., 2009, 96, 521-539.

14 S. L. Veatch and S. L. Keller, Phys. Rev. Lett., 2002, 89, 268101.

15 S. L. Veatch and S. L. Keller, Biophys. J., 2003, 85, 3074-3083.

16 S. L. Veatch, I. V. Polozov, K. Gawrisch and S. L. Keller, Biophys. J., 2004, 86, 2910-2922.
17 N. Bezlyepkina, R. S. Gracia, P. Shchelokovskyy, R. Lipowsky and R. Dimova, Biophys. J., 2013, 104, 1456-1464.

18 E. London, Acc. Chem. Res., 2019, 52, 2382-2391.

19 A. Kusumi, T. K. Fujiwara, R. Chadda, M. Xie, T. A. Tsunoyama, Z. Kalay, R. S. Kasai and K. G. N. Suzuki, Annu. Rev. Cell Dev. Biol., 2012, 28, 215-250.

20 F. Schneider, D. Waithe, M. P. Clausen, S. Galiani, T. Koller, G. Ozhan, C. Eggeling and E. Sezgin, Mol. Biol. Cell, 2017, 28, 1507-1518.

21 A. P. Liu and D. A. Fletcher, Biophys. J., 2006, 91, 4064-4070.

22 A. Honigmann, S. Sadeghi, J. Keller, S. W. Hell, C. Eggeling and R. L. C. Vink, eLife, 2014, 3, e01671.

23 K. Morigaki and Y. Tanimoto, Biochim. Biophys. Acta, Biomembr., 2018, 1860, 2012-2017.

24 M. I. Hoopes, R. Faller and M. L. Longo, Langmuir, 2011, 27, 2783-2788.

25 R. Parthasarathy, C.-h. Yu and J. T. Groves, Langmuir, 2006, 22, 5095-5099.

26 T.-Y. Yoon, C. Jeong, S.-W. Lee, J. H. Kim, M. C. Choi, S.-J. Kim, M. W. Kim and S.-D. Lee, Nat. Mater., 2006, 5, 281-285.

27 O. Purrucker, A. Förtig, K. Lüdtke, R. Jordan and M. Tanaka, J. Am. Chem. Soc., 2005, 127, 1258-1264.

28 F. Roder, O. Birkholz, O. Beutel, D. Paterok and J. Piehler, J. Am. Chem. Soc., 2013, 135, 1189-1192.

29 F. Okada and K. Morigaki, RSC Adv., 2015, 5, 1507-1513.

30 T. Okazaki, Y. Tatsu and K. Morigaki, Langmuir, 2010, 26, 4126-4129.

31 Y. Tanimoto, K. Okada, F. Hayashi and K. Morigaki, Biophys. J., 2015, 109, 2307-2316.

32 A. Janshoff and C. Steinem, Biochim. Biophys. Acta, 2015, 1853, 2977-2983.

33 N. Teske, J. Sibold, J. Schumacher, N. K. Teiwes, M. Gleisner, I. Mey and C. Steinem, Langmuir, 2017, 33, 14175-14183.

34 T. D. Lazzara, C. Carnarius, M. Kocun, A. Janshoff and C. Steinem, ACS Nano, 2011, 5, 6935-6944.

35 M. Gleisner, I. Mey, M. Barbot, C. Dreker, M. Meinecke and C. Steinem, Soft Matter, 2014, 10, 6228-6236.

36 K. Kumar, L. Isa, A. Egner, R. Schmidt, M. Textor and E. Reimhult, Langmuir, 2011, 27, 10920-10928.

37 A. Oshima, H. Nakashima and K. Sumitomo, Langmuir, 2019, 35, 11725-11734.

38 K. Sumitomo and A. Oshima, Langmuir, 2017, 33, 13277-13283.

39 H. Neubacher, I. Mey, C. Carnarius, T. D. Lazzara and C. Steinem, Langmuir, 2014, 30, 4767-4774.

40 G. W. de Groot, S. Demarche, M. G. Santonicola, L. Tiefenauer and G. J. Vancso, Nanoscale, 2014, 6, 2228-2237.

41 F. Heinemann and P. Schwille, ChemPhysChem, 2011, 12, 2568-2571.

42 K. Sumitomo, Y. Tamba, Y. Shinozaki and K. Torimitsu, Appl. Phys. Express, 2010, 3, 107001.

43 A. Orth, L. Johannes, W. Römer and C. Steinem, ChemPhysChem, 2012, 13, 108-114.

44 O. M. Schütte, I. Mey, J. Enderlein, F. Savić, B. Geil, A. Janshoff and C. Steinem, Proc. Natl. Acad. Sci. U. S. A., 2017, 114, E6064-E6071. 
45 M. Schwamborn, J. Schumacher, J. Sibold, N. K. Teiwes and C. Steinem, Analyst, 2017, 142, 2670-2677.

46 T. Baumgart, G. Hunt, E. R. Farkas, W. W. Webb and G. W. Feigenson, Biochim. Biophys. Acta, 2007, 1768, 2182-2194.

47 A. S. Klymchenko and R. Kreder, Chem. Biol., 2014, 21, 97-113.

48 E. Sezgin, I. Levental, M. Grzybek, G. Schwarzmann, V. Mueller, A. Honigmann, V. N. Belov, C. Eggeling, U. Coskun, K. Simons and P. Schwille, Biochim. Biophys. Acta, 2012, 1818, 1777-1784.

49 F. S. Ariola, Z. Li, C. Cornejo, R. Bittman and A. A. Heikal, Biophys. J., 2009, 96, 2696-2708.

50 S. L. Veatch and S. L. Keller, Phys. Rev. Lett., 2005, 94, 148101.

51 N. Kahya, D. Scherfeld, K. Bacia, B. Poolman and P. Schwille, J. Biol. Chem., 2003, 278, 28109-28115.

52 S. L. Veatch and S. L. Keller, Biochim. Biophys. Acta, 2005, 1746, 172-185.

53 N. Kahya, D. Scherfeld, K. Bacia and P. Schwille, J. Struct. Biol., 2004, 147, 77-89.

54 A. Pokorny, L. E. Yandek, A. I. Elegbede, A. Hinderliter and P. F. F. Almeida, Biophys. J., 2006, 91, 2184-2197.

55 O. M. Schütte, A. Ries, A. Orth, L. J. Patalag, W. Römer, C. Steinem and D. B. Werz, Chem. Sci., 2014, 5, 3104-3114.

56 S. J. Sahl, M. Leutenegger, M. Hilbert, S. W. Hell and C. Eggeling, Proc. Natl. Acad. Sci. U. S. A., 2010, 107, 6829-6834.

57 A. Honigmann, C. Walter, F. Erdmann, C. Eggeling and R. Wagner, Biophys. J., 2010, 98, 2886-2894.

58 C. Eggeling, C. Ringemann, R. Medda, G. Schwarzmann, K. Sandhoff, S. Polyakova, V. N. Belov, B. Hein, C. von Middendorff, A. Schönle and S. W. Hell, Nature, 2009, 457, 1159-1162.

59 B. Kubsch, T. Robinson, R. Lipowsky and R. Dimova, Biophys. J., 2016, 110, 2581-2584.

60 A. J. García-Sáez, S. Chiantia and P. Schwille, J. Biol. Chem., 2007, 282, 33537-33544.

61 W.-C. Tsai and G. W. Feigenson, Biochim. Biophys. Acta, Biomembr., 2019, 1861, 478-485.

62 T. M. Konyakhina and G. W. Feigenson, Biochim. Biophys. Acta, 2016, 1858, 153-161.
63 S. L. Veatch and S. L. Keller, Biophys. J., 2003, 84, 725-726. 64 E. Baykal-Caglar, E. Hassan-Zadeh, B. Saremi and J. Huang, Biochim. Biophys. Acta, 2012, 1818, 2598-2604.

65 J. Huang, J. T. Buboltz and G. W. Feigenson, Biochim. Biophys. Acta, Biomembr., 1999, 1417, 89-100.

66 M. M. Stevens, A. R. Honerkamp-Smith and S. L. Keller, Soft Matter, 2010, 6, 5882-5890.

67 D. Marsh, Handbook of lipid bilayers, CRC Press, Hoboken, 2nd edn, 2013.

68 V. D. Gordon, M. Deserno, C. M. J. Andrew, S. U. Egelhaaf and W. C. K. Poon, Europhys. Lett., 2008, 84, 48003.

69 J. W. Kuhlmann, I. P. Mey and C. Steinem, Langmuir, 2014, 30, 8186-8192.

70 S. Spindler, J. Sibold, R. Gholami Mahmoodabadi, C. Steinem and V. Sandoghdar, Nano Lett., 2018, 18, 5262-5271.

71 J. O. Rädler, T. J. Feder, H. H. Strey and E. Sackmann, Phys. Rev. E: Stat. Phys., Plasmas, Fluids, Relat. Interdiscip. Top., 1995, 51, 4526-4536.

72 M. J. Sarmento, M. Prieto and F. Fernandes, Biochim. Biophys. Acta, 2012, 1818, 2605-2615.

73 R. Lipowsky, T. Rouhiparkouhi, D. E. Discher and T. R. Weikl, Soft Matter, 2013, 9, 8438-8453.

74 R. Lipowsky, Biol. Chem., 2014, 395, 253-274.

75 S. Nehls and A. Janshoff, Biophys. J., 2017, 113, 1822-1830.

76 M. Kocun and A. Janshoff, Small, 2012, 8, 847-851.

77 R. Dimova, Adv. Colloid Interface Sci., 2014, 208, 225-234.

78 B. Kollmitzer, P. Heftberger, R. Podgornik, J. F. Nagle and G. Pabst, Biophys. J., 2015, 108, 2833-2842.

79 A. Callan-Jones, B. Sorre and P. Bassereau, Cold Spring Harbor Perspect. Biol., 2011, 3, a004648.

80 S. Maté, J. V. Busto, A. B. García-Arribas, J. Sot, R. Vazquez, V. Herlax, C. Wolf, L. Bakás and F. M. Goñi, Biophys. J., 2014, 106, 2606-2616.

81 M. J. Swamy, L. Ciani, M. Ge, A. K. Smith, D. Holowka, B. Baird and J. H. Freed, Biophys. J., 2006, 90, 4452-4465.

82 R. S. Petruzielo, F. A. Heberle, P. Drazba, J. Katsaras and G. W. Feigenson, Biochim. Biophys. Acta, 2013, 1828, 1302-1313.

83 P. Carravilla, J. L. Nieva, F. M. Goñi, J. Requejo-Isidro and N. Huarte, Langmuir, 2015, 31, 2808-2817. 